\title{
Neutrino burst from failed supernovae as a promising target of neutrino astronomy
}

\author{
Kohsuke Sumiyoshi* \\ Numazu College of Technology, Ooka 3600, Numazu, Shizuoka 410-8501, Japan \\ E-mail: sumi@numazu-ct.ac.jp \\ Ken'ichiro Nakazato \\ Department of Astronomy, Kyoto University, Kyoto 606-8502, Japan \\ E-mail: nakazato@kusastro.kyoto-u.ac.jp \\ Hideyuki Suzuki \\ Faculty of Science and Technology, Tokyo University of Science, Noda, Chiba 278-8510, Japan \\ E-mail: suzukiheph.noda.tus.ac.jp
}

\section{Shoichi Yamada}

Science and Engineering \& Advanced Research Institute for Science and Engineering,

Waseda University, Shinjuku, Tokyo 169-8555, Japan

E-mail: shoichi@heap.phys.waseda.ac.jp

\begin{abstract}
We study the unique characteristics of neutrino bursts from the black-hole-forming collapse of non-rotational massive stars, which is a definite branch among other core-collapse supernovae. We clarify that the neutrino signals from the failed supernovae are short and energetic, being different from the ordinary supernova neutrinos, by performing the numerical simulations of the general relativistic neutrino-radiation hydrodynamics with the neutrino reactions rates. Based on the calculated spectra of neutrino emission, we predict that the number of neutrino detection at the Super-Kamiokande is large enough to discuss the detailed differences of the neutrino signals using the sets of equation of state including hyperons and quarks. Therefore, the massive stellar collapse with the short neutrino burst is an important target of neutrino astronomy to probe the properties of dense matter.
\end{abstract}

11th Symposium on Nuclei in the Cosmos, NIC XI

July 19-23, 2010

Heidelberg, Germany

\footnotetext{
${ }^{*}$ Speaker.
} 


\section{Failed explosion from massive stars}

The gravitational collapse of massive stars is the fate of the stellar evolution, which leads to the variety of explosive phenomena, the formation of compact objects and the neutrino burst [1, 2]. While the supernova explosion is the outcome of the collapse and bounce from the massive stars of $\sim 20 \mathrm{M}_{\odot}$, the fate of non-rotating massive stars of $\sim 40 \mathrm{M}_{\odot}$ is the failed explosion since the progenitor is too massive. Since the iron core is too large, the propagation of shock wave is hindered by the intense accretion of material. Right after the core bounce, the nascent proto-neutron star at center becomes massive rapidly as the accretion proceeds. It soon collapses dynamically to the black hole when the mass reaches the critical mass. During the short evolution of the proto-neutron star from the birth to the re-collapse, a bunch of neutrinos is emitted from the accreting protoneutron star. The duration of neutrino emission is short since the emission is terminated when the black hole is formed.

We study the properties of such neutrino bursts from the black-hole-forming collapse of massive stars by performing the numerical simulations of the general relativistic neutrino-radiation hydrodynamics [3]. We examine systematically the unique characteristics of neutrino bursts by adopting the sets of the equation of state [4, 5, 6] and the progenitors [7]. We show that the duration of the neutrino bursts is short and it is sensitive to the softness of the equation of state. We explore the emergence of hyperons, pions and quarks in dense matter during the collapse and reveal the signature of the appearance of new degrees of freedom in the neutrino bursts. We further evaluate the number of neutrino detection at the Super-Kamiokande for a Galactic event $[8]$. We demonstrate that the neutrino burst from the failed supernovae is a new promising target of neutrino astronomy, which can identify the formation of black hole formation and probe the properties of dense matter [9].

\section{Neutrino-radiation hydrodynamics and EOS tables}

We perform the numerical simulations of the gravitational collapse of non-rotating massive stars to follow the dynamics starting from the initial collapse, the core bounce, the matter accretion, the birth of a proto-neutron star and its subsequent evolution up to the black hole formation. The numerical simulations are performed by the numerical code to solve the general relativistic neutrino-radiation hydrodynamics [10] with the neutrino reactions rates [11] under the spherical symmetry. Since we solve the Boltzmann equation, we can evaluate the neutrino flux and energy spectrum in detail to predict the properties of neutrino emission.

We adopt the profile of iron core inside the massive stars of $40-50 \mathrm{M}_{\odot}$ from the stellar evolutionary calculations as initial models. We present here a case of the $40 \mathrm{M}_{\odot}$ star by Woosley and Weaver [12]. We studied the dependence of neutrino characteristics on the progenitors and found that the density profiles of the progenitors (depending on stellar mass, metallicity and evolutionary models) may affect the evolution [7, 13].

In order to perform the numerical simulations of black-hole-forming collapse, the data set of equation of state (EOS) for extreme conditions is necessary. We utilize the tables of the equation of state based on the relativistic mean field theory with the extension to include hyperons, pions and quarks. The Shen-EOS is constructed by the relativistic mean field theory with the local density 

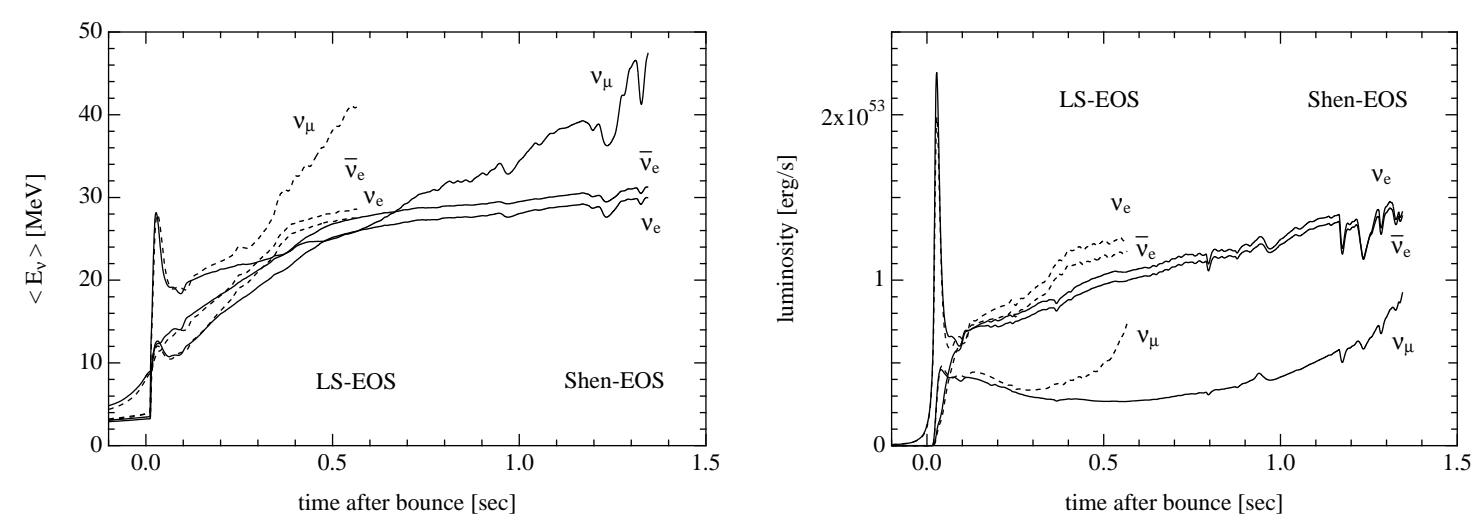

Figure 1: The time evolution of average energy (left) and luminosity (right) for LS-EOS and Shen-EOS.

approximation [14, 15]. Recently, the extension of Shen-EOS has been made to include hyperons in addition to nucleons (Hyperon-EOS) [16]. In addition, the extension of Shen-EOS table to the quark degrees of freedom has been done within the MIT bag model (Quark-EOS) [17]. The counter part, within the nucleon degree of freedom, of the Shen-EOS is the Lattimer-Swesty EOS (LS-EOS) [18], which is based on the extension of the compressible liquid drop model.

The maximum mass of cold neutron stars is $2.2 \mathrm{M}_{\odot}$ for Shen-EOS whereas it is $1.8 \mathrm{M}_{\odot}$ for LSEOS with a choice of the incompressibility, $\mathrm{K}=180 \mathrm{MeV}$. When we compare Hyperon-EOS with Shen-EOS, the mixture of hyperon softens the EOS and it reduces the maximum mass down to $1.6 \mathrm{M}_{\odot}$. In the case of Quark-EOS, the maximum mass is $1.8 \mathrm{M}_{\odot}$ for the case with the bag constant of $\mathrm{B}=250 \mathrm{MeV} / \mathrm{fm}^{3}$. This difference of the softness of EOS leads to the different time evolution of neutrino emission and is crucial to determine the duration of the neutrino burst. We compare here the properties of the neutrino bursts in the four cases with Shen-EOS, Hyperon-EOS, Quark-EOS and LS-EOS to see the influence of the EOS and the mixture of hyperons and quarks.

\section{Properties of short neutrino bursts}

We show in Fig. 1 the time evolution of the average energy (left) and luminosity (right) of neutrinos for the cases of Shen-EOS and LS-EOS. The average energies and luminosities for three flavors increase rapidly due to the temperature increase in the contracting proto-neutron stars. The neutrino bursts end up at $0.6 \mathrm{~s}$ and $1.3 \mathrm{~s}$ for LS-EOS and Shen-EOS cases, respectively, when the black hole formation occurs at the critical mass. Since the the critical mass of the accreting object is determined by the EOS, the duration of the neutrino burst reflects the softness of the EOS. Therefore, the neutrino burst from failed explosions can be a probe of the softness of dense matter.

We stress that the properties of the neutrino burst are different from those of the supernova neutrinos from ordinary explosions. While the supernova neutrinos last for $\sim 20 \mathrm{~s}$ from the quasihydrostatic proto-neutron star with the bright optical display of the supernova explosion, the neutrinos from the failed explosion last only for a short period until the black hole formation in the optically invisible event. The neutrinos from the failed supernovae are energetic with the increasing luminosities in the short period $(\sim 1 \mathrm{~s})$ in contrast to the exponential decay in the case of supernova neutrinos. 

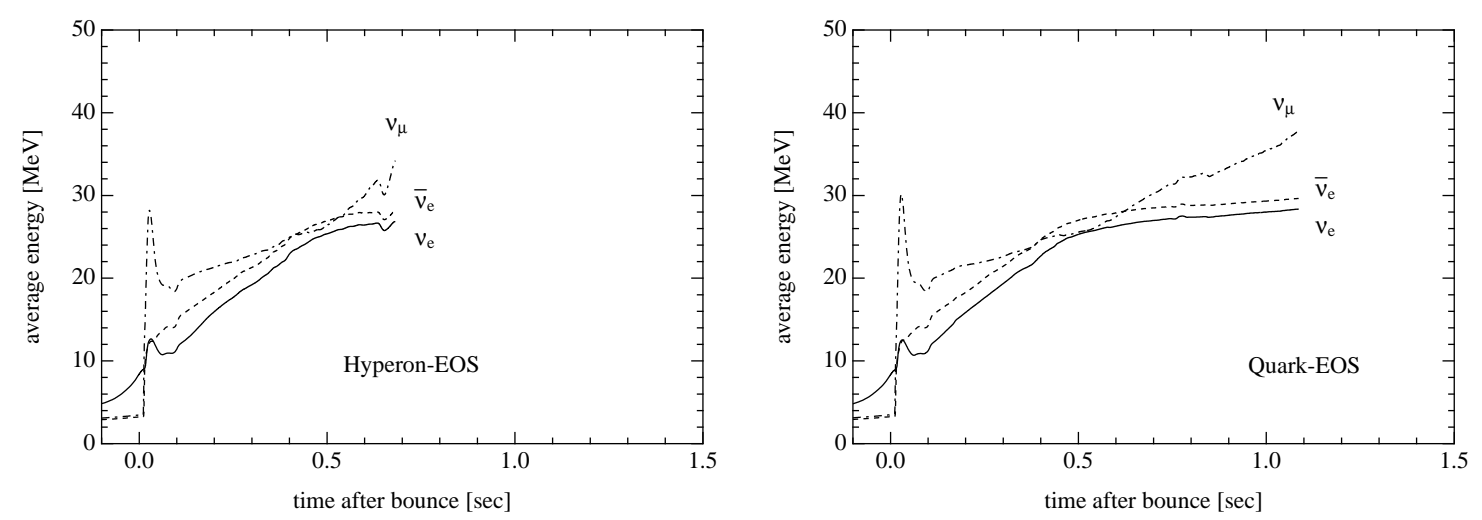

Figure 2: The time evolution of average energy for Hyperon-EOS (left) and Quark-EOS (right).

We show in Fig. 2 the time evolution of the average energy of neutrinos for the case of Hyperon-EOS (left) and Quark-EOS (right). In the case of Hyperon-EOS, the re-collapse to the black hole occurs at $0.7 \mathrm{~s}$ and the neutrino burst stops earlier than the case of Shen-EOS [5]. This timing is determined by the emergence of hyperons since the appearance of hyperons softens the EOS. In the case of Quark-EOS, the neutrino burst lasts for $1.1 \mathrm{~s}$, which is close to the case of ShenEOS [6]. This is because the quarks appear only at the final stage of the shrinking evolution of the massive proto-neutron star. The emergence of a new degree of freedom in dense matter shortens the neutrino burst and, therefore, may be probed by the observation of neutrino bursts in the failed explosions. In order to identify the appearance of exotics, it is important to distinguish them from the case of soft nucleonic EOS. As demonstrated in [9], the difference in the time evolution of neutrino energies can be used to discriminate the expected neutrino events between LS-EOS and Hyperon-EOS in spite of the similar short duration.

\section{Observational predictions}

Based on the numerical results of neutrino emission, we evaluate the number of neutrino detection at the Super-Kamiokande for a Galactic event by taking into account the neutrino oscillations [8]. We predict that the event number amounts to $\sim 10^{4}$ and is comparable to the case of supernova explosions. By the statistical analysis, we found that one can discriminate the detailed differences of the neutrino signals using the sets of equation of state including hyperons [9. We remark that the failed explosion can be popular as the ordinary supernovae in the estimate based on the population of massive stars [19], the observation of faint supernovae [2] and a black hole [20]. The planned survey of disappearance of massive stars [19] is helpful to obtain the detailed information on the progenitor, which may affect the duration of neutrino signal through the accretion rate. The construction of large neutrino detectors [21 22] may reveal the characteristics of neutrinos from the failed explosions inside the galaxies far away in future.

In summary, the massive stellar collapse with the short neutrino burst is an important astronomical target as a probe of the equation of state of the hot and dense matter. Having the systematic studies of the numerical simulations adopting the models of massive stars of $40-50 \mathrm{M}_{\odot}$ with our development of the data tables of the equation of state with hyperons, pions and quarks based on 
the Shen EOS, the future observation of the black-hole-forming collapse of the massive stars with the associated detection of the neutrino burst is awaited. Systematic studies regarding the dependence on various progenitors and the EOS by microscopic many body calculations are necessary to reduce the ambiguity in the neutrino signals.

\section{Acknowledgement}

K. Sumiyoshi is grateful to A. Ohnishi, C. Ishizuka, H. Shen, K. Oyamatsu and H. Toki for the fruitful collaborations on the EOS tables. This work is supported by the Grants-in-Aid for the Scientific Research $(18540295,19540252,20105004,22540296)$ of the MEXT of Japan. The numerical simulations were performed at the supercomputing resources at JAEA/Tokai, YITP (Kyoto University), RCNP (Osaka University), The University of Tokyo, KEK and CfCA in NAOJ.

\section{References}

[1] H. Suzuki, in: M. Fukugita, A. Suzuki (Eds.), Physics and Astrophysics of Neutrinos, Springer-Verlag, Tokyo, 1994, p. 763.

[2] K. Nomoto, N. Tominaga, M. Tanaka, K. Maeda, H. Umeda, AIP Conf. Proc. 937 (2007) 412.

[3] K. Sumiyoshi, S. Yamada, H. Suzuki, S. Chiba, Phys. Rev. Lett. 97 (2006) 091101.

[4] K. Sumiyoshi, S. Yamada, H. Suzuki, Astrophys. J. 667 (2007) 382.

[5] K. Sumiyoshi, C. Ishizuka, A. Ohnishi, S. Yamada, H. Suzuki, Astrophys. J. 690 (2009) L43.

[6] K. Nakazato, K. Sumiyoshi, S. Yamada, Astrophys. J. 721 (2010) 1284.

[7] K. Sumiyoshi, S. Yamada, H. Suzuki, Astrophys. J. 688 (2008) 1176.

[8] K. Nakazato, K. Sumiyoshi, H. Suzuki, S. Yamada, Phys. Rev. D78 (2008) 083014.

[9] K. Nakazato, K. Sumiyoshi, H. Suzuki, S. Yamada, Phys. Rev. D81 (2010) 083009.

[10] K. Sumiyoshi, S. Yamada, H. Suzuki, H. Shen, S. Chiba, H. Toki, Astrophys. J. 629 (2005) 922.

[11] S. W. Bruenn, Astrophys. J. Suppl. 58 (1985) 771.

[12] S. E. Woosley, T. Weaver, Astrophys. J. Suppl. 101 (1995) 181.

[13] T. Fischer, S. C. Whitehouse, A. Mezzacappa, F.-K. Thielemann, M. Liebendörfer, Astron. Astrophys. 499 (2009) 1.

[14] H. Shen, H. Toki, K. Oyamatsu, K. Sumiyoshi, Nucl. Phys. A637 (1998) 435.

[15] H. Shen, H. Toki, K. Oyamatsu, K. Sumiyoshi, Prog. Thoer. Phys. 100 (1998) 1013.

[16] C. Ishizuka, A. Ohnishi, K. Tsubakihara, K. Sumiyoshi, S. Yamada, J. Phys. G 35 (2008) 085201.

[17] K. Nakazato, K. Sumiyoshi, S. Yamada, Phys. Rev. D77 (2008) 103006.

[18] J. M. Lattimer, F. D. Swesty, Nucl. Phys. A535 (1991) 331.

[19] C. S. Kochanek, et al., Astrophys. J. 684 (2008) 1336.

[20] A. H. Prestwich, et al., Astrophys. J. 669 (2007) L21.

[21] S. Ando, J. F. Beacom, H. Yuksel, Phys. Rev. Lett. 95 (2005) 171101.

[22] M. D. Kistler, H. Yuksel, S. Ando, J. F. Beacom, Y. Suzuki, arXiv:0810.1959 (2008). 